\title{
Molecular dynamics study of the structure, flexibility and dynamics of thermostable L1 lipase at high temperatures.
}

\begin{abstract}
Molecular Dynamics (MD) simulations have been used to understand how protein structure, dynamics, and flexibility are affected by adaptation to high temperature for several years. We report here the results of the high temperature MD simulations of Bacillus stearothermophilus L1 (L1 lipase). We found that the N-terminal moiety of the enzyme showed a high flexibility and dynamics during high temperature simulations which preceded and followed by clear structural changes in two specific regions; the small domain and the main catalytic domain or core domain of the enzyme. These two domains interact with each other through a $\mathrm{Zn}(2+)$ binding coordination with Asp-61 and Asp-238 from the core domain and His-81 and His-87 from the small domain. Interestingly, the His- 81 and His- 87 were among the highly fluctuated and mobile residues at high temperatures. The results appear to suggest that tight interactions of $\mathrm{Zn}(2+)$-binding coordination with specified residues became weak at high temperature which suggests the contribution of this region to the thermostability of the enzyme.
\end{abstract}

Keyword: Thermostability; Thermoalkalophilic lipase; Molecular dynamics simulation; Protein flexibility. 